\title{
Posibilidades exploradas y por explorar del currículo
}

\section{Explored and unexplored possibilities of the curriculum}

\author{
ARRIETA, Adolfo ${ }^{1}$ \\ MONTES, Donicer, E. ${ }^{2}$ \\ HERNÁNDEZ, Darwin, Y. ${ }^{3}$
}

\begin{abstract}
Resumen
Este artículo explora las tradiciones curriculares más relevantes y como estas están asociadas a diferentes concepciones ideológicas que pretenden formar determinado tipos de seres humanos de acuerdo a las contingencias históricas y económicas de cada momento. También ofrece una perspectiva más abierta y flexible para concebir, desarrollar y evaluar un currículo como herramienta que empodera a los individuos hacia su auto-realización, la reflexión crítica, conciencia hacia las relaciones de poder, y su agenciamiento hacia equidad y solidaridad social para un mundo más democrático.

Palabras clave: concepción curricular, perspectivas del currículo, diseño curricular
\end{abstract}

\begin{abstract}
This article explore the most relevant curricular traditions and how they relate to certain ideological conceptions as well as how they aims to the formation of certain types of human beings according defined historical and economic contingencies. It also offer more open and flexible perspective to conceive, develop and assess a curriculum as tool to empower individuals towards their own selfauthorship, their critical reflection, awareness of power relations, and agency towards social solidarity and equity for a better democratic world.

key words: curriculum conception, curriculum perspectives, curriculum design
\end{abstract}

\section{Introducción}

La palabra currículo en la antigua Roma se usaba para denominar el recorrido que los carros de carrera hacían en la pista de competencia. Más adelante esta palabra fue empleada metafóricamente para expresar los cursos y contendidos que tenían que transitar los jóvenes en la escuela. Uno de los primeros trabajos fundamentales de currículo fue desarrollado por Bobbit (1918) quien asumió una perspectiva científica del currículo concebido como una seriede actividades que los niños y joven deben de experimentar para poder enfrenatar la vida adulta. En 1920, escribió el libro " como construir un currículo en donde presenta una serie de criterio para construir objetivos resultan relevate en nuestros días. Bobbit definió currículo como un instrumento de control o regulación para resolver los problemas sociales; sin emabago fue criticado por haber asumido un enfoque

\footnotetext{
${ }^{1}$ PhD en Ciencias de la Educación. Profesor Asociado. Departamento de Lenguas. Universidad de Sucre. Correo electrónico de contacto adolfo.arrieta@unisucre.edu.co

${ }^{2}$ PhD en Genética y Mejoramiento Genético Animal. Profesor titular. Departamento de Zootecnia . Universidad de Sucre. donicer.montes@unisucre.edu.co

${ }^{3}$ PhD en Ciencias Agrarias. Profesor asociado. Departamento de Zootecnia. Universidad de Sucre. darwin.hernandez@unisucre.edu.co
} 
conductista y efiecientista. En contraste Dewey (1902) concibió el currículo como una "continua reconstrucción de experiencias presentes de los niños que llegan a representaciones organizadas de verdades aceptadas denominadas estudios..." (pp. 11-12). Desde esta perspectiva el currículo para los niños se convierte en elemento mediador para del mundo que los rodea. Dewey consideraba que la educacion es proceso social a través del cual se puede construir una sociedad democrática. Consideraba que el desarrollo cognitivo y social de los niños podría darse a través de cualquier asigntura y que los estudiantes podrían desarrolar pensamiento científico a través de procesos de indagación racional.

Más tarde, Tyler (1957) definió currículo como un conjunto de experiencias de aprendizaje planeadas y direccionadas por la escuela para el logro de las metas educativas. Para ello propuso que los objetivos se deribaran de las necesidades de los estudiantes y la sociedad en genera; que a partir de estas se crearan actividades de aprendizaje y que las metas instruccionales debrian evaluase. En la misma línea, Taba (1962) contribuyó con una noción de currículo que permite caracterizar y determinar la funcionalidad de sus elementos constitutivos. De acuerdo a Taba un currículo está constituido de metas y objetivos, los cuales sugieren la selección y organización de contenidos que se debería orientar desde unos principios pedagógicos direccionados.

En 1968, Paulo Freire publico Pedagogia del Oprimido en donde amplia la pespectiva de la educacion como una herramienta de reflexión critica y de concientización social para contruir equidad social y democracia. Esta perspectiva critica de la educacion permitio asumir elcurriculo como una posibilidad de indagación critica de los problemas sociales desde una pedagogía basada en la solución de problemas y el desarrollo de pensamito critico para emancipación intelectual y social. En I misma línea, Pinar (1995) amplia el concepto de currículo resaltado que el currículo es necesario estudiarlo desde una perspectiva histórica, política, racial femonenologica, postmodernista e internacional asumiendo un enfoque mutidisciplinar. En ese sentido, el objetivo de este estudio fue explorar bajo la metodología de revisión crítica de literatura, las posibilidades exploradas y por explorar del currículo como estrategia de conocimiento complementar a aplicar en la formación profesoral.

\section{Metodología}

En este estudio se seleccionaron 30 artículos seminales y 15 libros sobre diseño curricular diseños curriculares escritos en los últimos 15 años. Se emplearon varias bases de datos tales como Redalyc, Scopus, Scielo Science Direct y Springer entre otras, siguiendo la estructura planteada por Gómez-Gómez, Danglot-Banck y VelásquezJones (2001). Los criterios de selección fueron los siguientes: clasificación de artículos por relevancia temática de los diseños, fundamentos ideológicos que justifican los diferentes tipos de diseño curriculares, características de los mismos, índice de citación de los mismos en Google académico y relevancia de los mismos en el contexto educativo, sociocultural y político. Para el análisis del mismo se realizó un proceso analítico de lectura profunda identificando líneas temáticas a partir de proceso de análisis de contenido temático con matrices de revisión con dos macro líneas temáticas curriculares: posibilidades exploradas del currículo y posibilidades curriculares por explorar.

\section{Resultados}

\subsection{El currículo como posibilidad explorada}

Taba (1962) planteó la enseñanza de los contenidos desde el razonamiento inductivo a través de estrategias pedagógicas que les permitiera a los estudiantes formar conceptos, interpretar información y aplicar principios. Muchas de estas concepciones curriculares guiaron los diseños curriculares de los dos primeros tercios del siglo XX. Por ejemplo, la concepción de Dewey (1902), de naturaleza desarrollista, impacto los diseños curriculares ciclo escolar primario. En contraste, Taba (1962) y Tyler (1957) influyeron más en los diseños curriculares de los ciclos de educación básica, media y universitario. 
Otra perspectiva curricular surgió con Stenhouse (1984) quien definió currículo como un intento de comunicar los principios esenciales y característicos de una propuesta educativa de tal forma que esté abierta al escrutinio crítico público y pueda ser efectivamente traducida hacia la práctica. Este autor concibió el currículo como un proceso de integración continuo entre profesor, estudiante y conocimiento. Igualmente, contempla los contendidos y los medios son desarrollados desde el trabajo consensuado entre profesores y estudiantes, lo que conlleva a la construcción de significado. Desde esta perspectiva, el currículo se centra en el proceso de aprendizaje de los estudiantes. Fue Stenhouse (1984) quien posicionó al profesor como un estudioso y desarrollador de experiencias curriculares rompiendo la hegemonía prescriptiva de los expertos curriculares. Además, puso en tela de juicio las relaciones de poder entre expertos en desarrollo curricular y profesores y entre profesores y estudiantes. Esta nueva perspectiva curricular abrió nuevas posibilidades de agenciamiento curricular colaborativos entre docentes ofreciendo la posibilidad de escrutarlo críticamente y hacerlo más pertinente.

La concepción crítica de currículo surge por trascender la relación entre educación y sociedad. Su principal propósito radica en formar ciudadanos para convivir y trabajar en sociedad. Muchos autores tales como Apple (1995); Bourdieu (1967); Giroux (1981) comenzaron a cuestionar la relación entre escolarización y estado desde una posición crítica hacia la educación, el currículo y los procesos educativos. Esta perspectiva crítica buscó analizar las ideologías que subyacen en el proceso de escolarización en el estado moderno, las formas de reproducción de conocimiento, las relaciones de poder que se dan en el mismo y la forma como el sistema educativo se convierte en una herramienta de transmisión ideológica que permite la reproducción hegemónica de los sistemas de poder.

La teoría crítica busca emancipar a los individuos a través de la crítica ideológica sustentada en gran parte en el razonamiento dialectico. Esta teoría sentó sus fundamentos a través la escuela de Frankfort (1922) en los trabajos realizados por Horkheimer, Marause, Adorno y posteriormente Habermas la cual promueve la emancipación a través de la creación de concientización de las clases sociales (Vila, 2011). Desde esta teoría, el currículo se problematiza como un proceso que trasciende las nociones dicotómicas de las concepciones de currículo centradas en principios prácticos y técnicos de educación. De igual modo, cuestiona la forma cómo los currículos median y perpetúan las relaciones de poder hegemónicas existentes. La teoría crítica resalta que el currículo no es un proceso neutral sino un proceso político e ideológico que trata de mantener el estatus quo para muchos y preservar el poder de las elites. Igualmente, busca develar las contradicciones inherentes a los currículos y las formas de resistencias democráticas que hagan del mismo no un proceso de adoctrinamiento sino un proceso emancipador.

Dentro de la perspectiva crítica Freire $(2006$; 2009) posiciona la teoría critica desde de la praxis en donde convergen la reflexión, el diálogo y la acción como procesos de desconstrucción ideológica y agenciamiento de construcción de una sociedad democrática. El principal propósito de la educación emancipadora es empoderar individuos dentro de las comunidades para construir procesos sociales más equitativos que posibiliten la transformación de sus condiciones de vida. Este proceso de transformación surge de la creación de procesos de concientización y solución de problemas a través de ciclos continuos de reflexión dialogo y acción para develar y emancipar a los individuos y las comunidades de las ideologías que los oprimen. En este sentido la teoría critica transciende la reflexión y se convierte en un proyecto de intervención en donde educadores y educandos coconstruyen acciones políticas, éticas y culturales para una sociedad más equitativa y participativa (Giroux, 2013). Desde la concepción crítica del currículo pueden surgir propuestas curriculares en donde la comunidad educativa pueda ser agente de su momento histórico y asumir posiciones y acciones democráticas hacia la solución de los problemas sociales que enfrenta el siglo XXI. 


\subsection{El currículo e ideología}

Las concepciones curriculares arriba presentadas representan cuatro perspectivas ideológicas diferentes de currículo (Schiro, 2013): la ideología académica de profesores, la ideología de la eficiencia social, la ideología centrada en el estudiante, y la ideología de la reconstrucción social. La ideología académica tiene como propósito transmitir conocimiento a los estudiantes universitarios organizado en disciplinas Esto implica, un currículo basado en el aprendizaje de contenidos y las formas de pensar de las disciplinas las cuales se sustentan en la diseminación de conocimiento verdadero. En este tipo de currículo los académicos se convierten en mediadores del conocimiento producido por comunidades científicas y los estudiantes asumen un rol de receptores.

La ideología de la eficiencia social apunta a entrenar a los estudiantes para satisfacer las necesidades de la sociedad en términos de entrenar a los jóvenes en habilidades y procedimientos que demanda el mercado laboral. Desde esta ideología, la instrucción prima sobre el conocimiento ya que busca medir en los individuos la experticia y las habilidades para el mercado laboral.

Por otro lado, la ideología centrada en el estudiante, tiene como propósito potenciar el desarrollo intelectual, social, emocional y físico de cada individuo. Las necesidades, intereses y falencias de los individuos son integrados a la planeación y procesos curriculares para potenciar sus conocimientos, habilidades y actitudes. La interacción entre los individuos y el medio crea procesos intersubjetivos de construcción de significados que potencializan la autonomía y la autorrealización.

La ideología centrada en la reconstrucción social, busca empoderar a los estudiantes hacia la transformación de una sociedad más equitativa y justa desde procesos de emancipación de las hegemonías que detentan el poder. El currículo es concebido como una herramienta ideológica que posibilita formas más simétricas de negociones de poder y transformación social (Giroux, 1981).

Las ideologías y sus respectivos tipos de currículos han surgido para responder a momentos históricos que han exigido la formación de determinados tipos de ciudadanos. Estas perspectivas de currículos, han ido acumulando progresivamente puntos de encuentro y desencuentro con respecto a los propósitos ideológicos arriba mencionados y los tipos de currículos que se desprenden de dichas ideologías. Por ejemplo, a nivel universitario, los diseñadores de currículo, los ejecutores de currículo y evaluadores de currículo deberían tomar decisiones con respecto al énfasis de formación, ya sea en la formación disciplinar o en la formación para el trabajo. Generalmente, los diseñadores y evaluadores de currículo apuntan hacia propósitos funcionales mientras que los profesores o ejecutores del currículo tienen a favorecer más la formación disciplinar. No obstante, la tendencia de la educación superior en las dos últimas décadas ha sido responder a sistemas de 'accountability' o controles de calidad, que buscan desarrollar competencias que respondan a las necesidades del mercado. Con este tipo de currículo se busca modificar los individuos para producir servicios, productos y explotar commodities.

El reto en nuestros tiempos es diseñar currículos que trasciendan los propósitos instrumentalistas heredados del modernismo y crear propuestas curriculares más posmodernistas, orgánicas y dinámicas que puedan responder a las complejidades que demanda la ciencia, la sociedad y sobre todo que le permitan a los individuos ser coconstrutores de conocimiento desde la ciencia para la sociedad y desde ellos para la sociedad y la ciencia.

\subsection{Una perspectiva posmodernista del currículo}

Doll (1993) propone un diseño desde una perspectiva posmodernista curricular centrado en el estudiante. Para este autor, un currículo crea y potencia experiencias ricas, recursivas, relacionales, rigurosas, e integradoras. Un currículo se caracteriza por ser rico porque es abierto posibilita la exploración profunda y amplia de construcción de significados desde procesos dialógicos entre los participantes que los lleven a explorar nuevas perspectivas desde complejos procesos de aprendizaje profundos (Biggs, 1999) y desde patrones mentales abiertos (Dweck, 
2008) que les posibilite convertir retos en oportunidades para desarrollar competencias relevantes para el mundo globalizado y el contexto local.

La segunda característica del currículo es su recursividad, es decir, que les provea a los estudiantes oportunidades frecuentes de revisitar y examinar reflexivamente como el conocimiento trasforma sus competencias académicas, sociales, éticas profesionales y científicas. El concepto de recursividad curricular, se contrapone al concepto de linealidad curricular, que concibe el aprendizaje como un proceso seriado acumulador de disciplinas de conocimientos el cual es más propio de los currículos académicos y tecnológicos. En contraste, la recursividad curricular, considera que el aprendizaje es un proceso activo, social, reciproco y dialógico que transforma a los individuos y el conocimiento. La recursividad es más inherente a los currículos centrados en el estudiante y al currículo de naturaleza socio-crítica.

La tercera característica de un currículo posmodernista es su naturaleza racional en donde las partes constitutivas del mismo se interrelacionen de manera flexible y elástica. Esta relación debería de darse desde una perspectiva orgánica, es decir, desde la interrelación de conocimientos, disciplinas e intenciones particulares de comprender y crear múltiples perspectivas de conocimiento.

La cuarta característica, es que todo currículo desde la perspectiva posmoderna es riguroso. Un currículo riguroso debería crear espacios y oportunidades de aprendizaje que posibilite experiencias de aprendizaje integrales las cuales pueden estar diseñadas desde enfoques de aprendizaje centrado en problemas, enfoques centrado en indagación, o centrado en proyectos que enfaticen el desarrollo del pensamiento crítico, la creatividad, la flexibilidad y la autonomía de los estudiantes (Doll, 1993).

\subsection{Currículo desde una perspectiva integral}

La posición ideológica posmodernista tiende a considerar el currículo desde una perspectiva integral. Un currículo integral busca hacer conexiones a través de las disciplinas al mundo real y usa el contexto local para crear oportunidades de aprendizaje (Drake \& Burns, 2004). Esta clase de currículo concibe el aprendizaje y la enseñanza como un proceso holístico e interactivo que refleja lo que sucede en el mundo real (Perez, 2008). Este modelo busca planear experiencias de aprendizaje que les permita a los estudiantes experimentar relaciones entre conceptos, disciplinas y sistemas con el mundo real. En un currículo, estas relaciones usualmente ocurren cuando se combinan asignaturas, relaciones conceptuales, unidades temáticas de varias disciplinas, trabajos colaborativos y apreciaciones crítica intertextuales para la solución de problemas. Drake y Burns (2004) proponen tres posibilidades de integración curricular:

Figure 1

Tipos de Enfoques de Currículo Integrado adaptado de Drake \& Burns (2004)

\begin{tabular}{|c|c|c|}
\hline Enfoque & Definición & Características \\
\hline Multidisciplinar & $\begin{array}{c}\text { Desarrolla un tema desde la perspectivas de varias } \\
\text { asignaturas. La integracion tematica puede ser parcial } \\
\text { o total. }\end{array}$ & $\begin{array}{c}\text { Se enfoca en el desarrollo de conceptos y } \\
\text { habilidades a traves de asignaturas. }\end{array}$ \\
\hline Interdisciplinar & $\begin{array}{c}\text { Se integran subdisciplias dentro de un area como } \\
\text { historia, geografia y ecomonia en ciencias sociales. }\end{array}$ & $\begin{array}{c}\text { Se contrastan conceptos y habilidades a } \\
\text { traves de varias disciplinas. }\end{array}$ \\
\hline Transdiciplinar & $\begin{array}{c}\text { Se organiza el curriculo a traves de experiencias de } \\
\text { aprendizaje comunes en las asignturas para desarrollar } \\
\text { relaciones teroricas y habilidades. }\end{array}$ & $\begin{array}{c}\text { Ofrece una comprencion sistemica de los } \\
\text { problemas y las disciplinas posibilitando a los } \\
\text { estudiantes plantear socluciones complejas y } \\
\text { cercanas a los problemas de la vida real. }\end{array}$ \\
\hline
\end{tabular}


Una opción de modelo multidisciplinar de unidades curriculares integrado se puede crear tomando temas globales sociales o industriales y desde aquí formular preguntas esenciales la que a su vez se subdividen en preguntas claves del tema o problema particulares de los estudiantes, con estas preguntas el profesor establece secuencias curriculares a partir de las preguntas y sub-preguntas apoyándose con expertos. Asimismo, los profesores, asesores y expertos hacen acompañamiento personalizado a los estudiantes creando convergencia entre lo académico, lo productivo y lo social. Al final, todos se convierten en evaluadores del proceso y el producto de los proyectos (Clayton, 2010). La siguiente grafica muestra la panorámica del proceso:

Figure 2

Modelo de Unidad Curricular multidisciplinar Integrada adaptado de (Clayton, 2010)
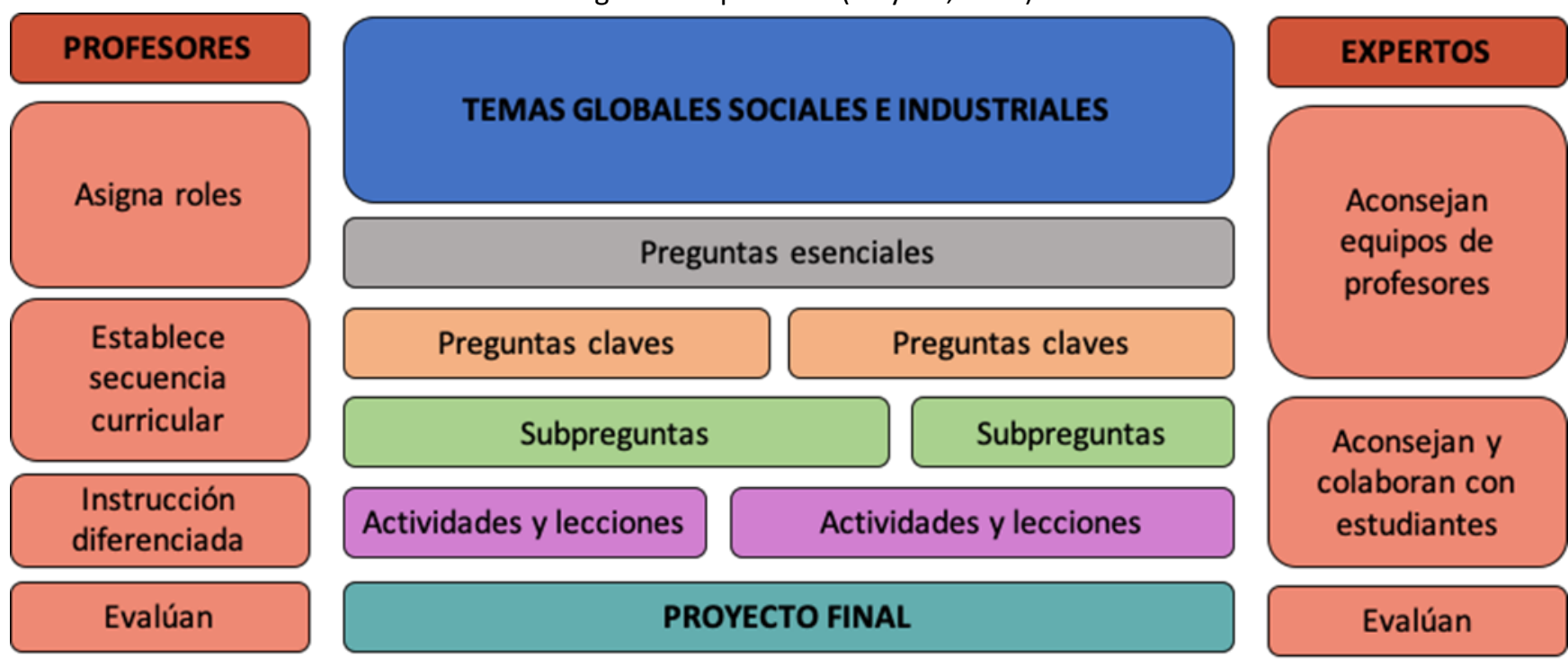

Es importante señalar que en este modelo multidisciplinar de unidades curriculares integrado subyacen posicionamientos ideológicos eficiencias ya que en gran medida los fines del modelo tributan a la formación de individuos para el mercado a través de estándares y participación de los expertos procedente del sector industrial quienes se convierten en elementos mediadores de la eficiencia y la formación técnica denominada el nuevo taylorismo (Au, 2011). En otras palabras, el currículo y la educación se convierten en instrumentos hegemónicos al servicio de los intereses económicos multinacionales los cuales usan la educación como medio para tributar a sus intereses económicos.

\subsection{El currículo como posibilidad de explorar}

El propósito de los diseños curriculares del siglo XXI debería trascender las estructuras jerárquicas centradas solo en fines mercantiles para aspirar a crear espacios de exploración de posibilidades de construcción creadora de democracia, justicia y equidad social. Para ello se requiere que los currículos ofrezcan condiciones de diversidad interna que fomenten contingencias que puedan emerger de dicha diversidad. En este sentido el currículo no solo debería responder a las particularidades de cada contexto y cómo esas particularidades están interrelacionadas entre los individuos y sociedad sino también contribuiría a potencializar la construcción de identidades desde la negociación de significados desde las subjetividades de los individuos. Al respecto Kegan (1982) subraya que llegar a ser una persona es una actividad de construcción de significados y que en todas las actividades de los seres humanos, sean estas sentimientos, pensamientos, percepciones o experiencias, median procesos de construcción de significados. En todo proceso de construcción de significado la relación de sujeto y 
objeto puede conllevar la auto-autoría de los individuos lo que a su vez potencia creatividad y diversidad interna dándole vida al currículo.

De igual modo, el currículo debería de fortalecerse desde la redundancia interna que permiten procesos de duplicación de aspectos similares o diferentes que produzcan posibilidades de redundancia y propendan por la coherencia expansiva del mismo desde la interacción de los individuos en el currículo y la interacción de las disciplinas para la creación de posibilidades de nuevas formas de conocimientos y de acciones consecuentes que permitan integrar y crear nuevas experiencias.

Además, el currículo tendría que imaginarse, gestionarse y evaluarse desde la interacción disciplinar la cual como se dijo arriba se puede hacer desde una perspectiva multidisciplinar, interdisciplinar o transdisciplinar. La interacciones disciplinares propician oportunidades para generar conocimientos con perspectivas más holísticas y le permite a los individuos enfrenar los problemas de la vida real de manera más creativa y flexible.

Asimismo, el currículo debería concebirse desde un control descentralizado para crear instancias de posibilidades de co-construcción de conocimiento colectivas y colaborativas que potencialicen la producción colectiva de conocimiento (Davis \& Sumara, 2008). En este periodo histórico la producción colectiva de conocimientos se da desde las comunidades de aprendizaje las cuales se organizan y auto-digieren en torno a intereses comunes y metas compartidas que facilitan la autorrealización, el sentido de pertinencia y el agenciamiento colaborativo de conocimiento. En resumen, el currículo visto desde las conciencias de diversidad interna, la redundancia interna, la interacción disciplinar y el control descentralizado crea ventanas de posibilidades de currículos que potencien no solo la reproducción sino también y producción de conocimiento.

Lo anterior implica una concepción dinámica y retadora del currículo. Dinámica porque, posibilita la mejor evolución de los procesos de desarrollo curricular a partir de procesos consensuados de co-construcción curricular desde espacios de diálogos y debates colaborativos de profesores, estudiantes y diferentes estamentos sociales. Retadora porque, usualmente las decisiones curriculares usualmente implican tensiones y conflictos entre los diferentes actores del proceso. Sin embargo, esta misma tensión es la que abre posibilidades de negociación y discusión convirtiendo los procesos de diseño, desarrollo y evaluación curricular en un ejercicio continuo y democrático de construcción de currículos significativos y pertinentes. En este sentido, el currículo se concibe como un proceso dinámico y en constante evolución en donde las metas del mismo responden y resinifican creativamente a las dinámicas de los procesos sociales, económicos, culturales y científicos.

Para responder a las permanentes tensiones que demanda este mundo globalizado y complejo es fundamental que los currículos sean flexibles, dinámicos, provisionales y contingentes. En este mundo globalizado se hacen necesario glocalizar los currículos desde la mediación dialógica de saberes locales y globales que posibiliten la interpretación crítica de las hegemonías circundantes. Asimismo, se requiere favorecer iniciativas de transversalidad curricular asumiendo una perspectiva más maleable del mismo en donde se celebre la diversidad cultural, lingüística y cognitiva de la comunidad educativa. En este contexto, el profesor asume un rol de creador de oportunidades contextualizadas de aprendizaje en donde los estudiantes se convierten en agentes constructores de su propio aprendizaje. Es decir, los estudiantes asumen el aprendizaje como un proceso exploratorio, contingente y relevante para el desarrollo de sus propias identidades. La evaluación se asume como un proceso formativo, continuo sustentado en criterios establecidos que mejoren los procesos de razonamiento, el uso del conocimiento, el respeto por la diversidad sociocultural y el desarrollo de pensamiento crítico de estudiantes.

Los currículos posmodernos se caracterizan por estar en continuo procesos de resignificación, comprensión, y transformación creativa de las realidades sociales del momento. Los currículos de esta era posmoderna pueden posibilitar espacios de comprensión profunda de nuestra realidad social hacia la reconstrucción una sociedad 
global que propenda por la justicia social, la compasión y la sostenibilidad ecología que les permita a los educadores fomentar procesos significativos y creativos que favorezcan una cultura de responsabilidad moral. Este tipo de currículo invita y celebra narrativas personales, alternativas personal, maestras desde el colorido de voces y los saberes que sus participantes. El anterior refleja una perspectiva compleja caótica y calidoscópica currículo el cual se según Briggs (1992) se puede concebir como una interrelación compleja de elementos que no pueden ser reducidos cerealistamente sino que hacen parte de un procesos sistemático global interdependiente como él lo señala a continuación: “Fenómenos naturales complejos como el clima no se puede quitar, limpiar y estudiar bajo vidrio en un laboratorio. Un árbol individual es el resultado de un vasto y cambiante conjunto de circunstancias únicas, un caleidoscopio de influencias como la gravedad, los campos magnéticos, la composición del suelo, el viento, los ángulos solares, hordas de insectos, cosecha humana, otros árboles, [y] fuerzas activas continuas, con demasiado hechos numerosos para determinarlos en detalle"(p. 14).

Todas las perspectivas curriculares deberían concebirse como potencialidades meta-teóricas que permitan la reflexión crítica de los currículos y que posibiliten reconstruir las intencionalidades ideológicas que los dinamizan hacia la perpetuación de relaciones de poder asimétricas y hegemónicas y que se abran a más posibilidades de construir y usar el conocimiento como elemento de mediación de solidaridad social y bien común. En otras palabras todo currículo es una herramienta de poder que se define institucionalmente, pero los profesores desde su reflexión crítica (Gimeno Sacristán, 1991; Brookfield, 1995) puedan agenciar currículos vivos que potencialicen la equidad, el espíritu crítico, la participación democrática, la identidad personal e identidad nacional.

En nuestro ejercico de poner en practica las posibilidades por explorar del currículo se ha realizado dos intervenciones curriculares que han posibilitado la resignficacion de los procesos curriculares a partir de procesos de investigacion acción con la comunidad y las escuelas. El primero desarrollado en un sector rural indígena de la región Caribe Colombiana en donde se resignfico el currículo desde la integración del capital cultural de la comunidad de San Antonio de Palmito en el departamento de Sucre a través de las herramientas de mediación y procesos de mediación a algunas asignturas de la maya curricular de la instucion educativa de San Antonio de Palmito. Los resultados de este estudio mostraron que la principal herramienta mediadoa de la comunidad era el proceso de tejido del Sombrero Vueltiao el cual se convierte en el artefacto que le da sentido y construye sentido a la comunidad y se resignfica como en el elmento identitario de la misma. Este curriuclo se construyo como un proceso colaboarivo con los estudiantes sus padres y la institución educativa en este sentido se rompió con la concepción gerarquica de currículo. El proceso permitio crear espacio democráticos de diálogos de de reconocimiento entre estudiantes y padres del sector urbano y rural del municipio. Las particularidades del contexto se convirtieron en herramienta mediadora del currículo desde una perspectiva interdisciplinar del ingles, el epanol y ciencias. Los estudiantes se convirtieron productores de conocimeinto al crear procesos crear productos multimediales que describían sus entorno ecológico y cultural. Igualmente empodero a los estudiantes a expresar desde sus voces su identidad cultural a través del currículo( Jaraba \& Arrieta,2012) .

La experiencia curricular exploarda surgio a partir de el poco nivel de compromiso y motivación de un grupo de estudiantes por aprender ingles en la escuela publica en Sincelejo, Sucre. En esta experiencia de trasformación curricular desde el aula. Se busco desarrollar procesos de afirmación de valores democráticos a través de experiencias de aprendizaje activo de los estudiantes y socialización de proyectos relacionacionados con el medio ambiente, cuidando mi salud usando valores como la responsabilidad, el autocontrol, el respeto y lagratitud. En este proyecto, los estudiantes reflexionaron críticamente acerca de los problemas de medio ambiente que había en sus barrios y ciudad, identificaron problemas y ofrecieron posibles soliciones a los mismos a través de proyectos que socializaron un numero signficativo de estudiantes en la isntutucion. A través de este proyecto los estudiantes desarrollaron compromiso social, instancias de justicia social, autonomía al invesitgar y proponer solución a probelams reales y expresar a través de sus voces lo que pensaban sobre las temáticas exploradas( Hernandez, Arrieta \& Rosado 2021 proxmo a publicar). En esta experiencia curricular se pueden aprenciar 
también elementos similares sobre los elementos dinamizadores de psobilibidades de currículos mas postmodernos en donde la relación de estudiantes con su objeto de aprendizaje lo lleve a ser autoautor de su proceso de aprendizaje y laforezca la co-construccion de conocimeinto relevante para sus vidas que propendan por la afirmación de valores democráticos, conciencia de justicia social y redudancia de coherencia expansiva entre los estudiantes, su contexto a través de expericias signficativas curriculares.

\section{Conclusiones}

En este artículo se han explorado las más relevantes perspectivas de las concepciones y diseños curriculares que han permitido configurar los procesos educativos en diferentes niveles educativos. La perspectiva de orientación técnica cuyo principal propósito es lograr un control intelectual y técnico del mundo enfatizando en la eficiencia y la certeza y que se sustenta en una concepción del conocimiento desde lo empírico desde posiciones epistémicas objetivas del conocimiento sustentado en teorías de naturaleza conductista, funcionalistas y sistemáticas. Pasando por una perspectiva de orientación interpretativa cuyo principal interés construir procesos de comprensión desde la intersubjetividades de los individuos y las maneras como estos construyen significados con otros sustentados en presupuestos teóricos constructivistas sustentados en la fenomenología del conocimiento, etnometodología, la lingüística y la interpretación hermenéutica de la realidad social. Atravesado la orientación critica que pretende conectar al hombre con su ser y el mundo social con el propósito de mejorar las condiciones de la vida de los humanos y develar los iniquidades sociales para promover la justicia social. Aquí la reflexión crítica juega un papel crucial ya que permite a los individuos llegar a la praxis con el propósito de mejorar la vida de los individuos. Esta concepción curricular esta sustentadas en la teoría critica, la teoría feminista, y el psicoanálisis. Llegando a una concepción curricular compleja que concibe el currículo desde una perspectiva ecológica en donde el aprendizaje se conciba como un proceso emergente y ecológico de los individuos en la sociedad. En este tipo de currículo como una interrelación de sus elementos constitutivos y el todo trascienda la suma de sus partes. En este sentido el currículo es dinámico, relacional, recursivo y agenciado por sus participantes. Desde estas perspectivas se puede analizar las orientaciones curriculares de programas desde ideologías subyacentes en los currículos, el énfasis que asumen los mismos y la evaluación de su pertinencia para el tipo de ser humano que e quiere formar en este mundo globalizado complejo y en constante cambio.

\section{Referencias bibliográficas}

Apple, M. (1995). Education and Power. New York and London: Routledge .

$\mathrm{Au}, \mathrm{W}$. (2011). Teaching under the new Taylorism: high-stakes testing and the standardization of the 21st century curriculum. Journal of Curriculum Studies, 43(1), 24-45.

Biggs, J. (1999). Teaching for quality learning at university. Open University Press, 165-203.

Bobbit, F. (1918). The Curriculum . Boston: Houghton Mifflin.

Bobbit, F. (1924). How to make a curriculum. Boston: Houghton Mifflin.

Bourdieu, P. (1967). Systems of Education and Systems of thought. International Social Science Journal, 19(3), 367-388.

Briggs, J. (1992). Fractals: The Pattern of Chaos - a New Aesthetic of Art, Science, Nature. New York: Simon \& Schuster.

Brookfield, S. (1995). Becoming a critically reflective teacher. San Francisco: Jossey Bass. 
Clayton, M. (2010). Designing multidisciplinary integrated curriculum units . Sacramento, California: The California Center for College and Career.

Davis, B., \& Sumara, D. (2008). Complexity as a theory of education. Transnational Curriuclum inquiry, 2, 33-44. Dewey, J. (1902). The Child and the Curriculum. Chicago : University of Chicago Press.

Doll, W. J. (1993). A Post-Modern Perspective on Curriculum. New York: Teachers College Press.

Drake, S., \& Burns, R. (2004). Meeting Standards Through Integrated Curriculum. Alexandria, Va: ASCD.

Dweck, C. (2008). Mindset: The New Psychology of Success. New York : Balntine books.

Freire, P. (2009). La Educacion como Practica de la Libertad. Mexico : Siglo XXI Espana .

Freire, P. (2006). Pedagogía del oprimido. Mexico: Siglo XXI.

Gimeno Sacristán, J. (1991). El curriculum: una reflexión sobre la práctica. Madrid : Ediciones Morata .

Giroux, H. (1981). Ideology, Culture and the Process of Schooling. Philadelphia, USA: Temple University Press .

Giroux, H. (2013). Una pedagogía de la resistencia en la edad del capitalismo de casino. Con-Ciencia Social, 17, 55-71.

Gómez-Gómez, M., Danglot-Banck, C., \& Velásquez-Jones, L. (2001). Bases para la revisión crítica de artículos médicos. Revista Mexicana de Pediatría, 68(4), 152-159.

Jaraba Ramírez, Diana Luz, \& Arrieta Carrascal, Adolfo. (2012). ETNOENGLISH: TRENZANDO PALABRAS, HISTORIAS Y PROYECTOS DE VIDA DEL PUEBLO ZENÚ. Íkala, Revista de Lenguaje y Cultura, 17(1), 95-104. Retrieved September 27, 2020, from http://www.scielo.org.co/scielo.php?script=sci_arttext\&pid=S012334322012000100007\&lng=en\&tlng=es.

Kegan, R. (1982). The Evolving Self: Problem and Process in Human Development. Cambridge, MA: Harvard University Press.

Perez, M. (2008). Curriculum Integral como trayecto de desarrollo en Abrahan Magendzo. Educación y Humanismo (15), 115-125.

Pinar, W.F., Reynolds, W.M., Slattery, P. And Taubman, P.M. (1995) Understanding Curriculum, New York, Lang.

Schiro, M. (2013). Curriculum theory. Thousand Oaks, California : Sage.

Stenhouse, L. (1984). Investigacion y desarrollo de curriculum . Espana : Morata .

Taba, H. (1962). Curriculum Development. Theory and Practice . New York : Harcourt, Brace and World .

Taylor, F. (1988). Principios de administracion cientifica . Mexico : Herrero Hermanos.

Tyler, R. (1957). The Curriculum then and now. Procedings of the 1956 Invitational Conference on Testing Problems. Princeton NJ: Educationa Testing Service.

Vila, D. (2011). Las concepciones de curriculum y su importancia en la elaboración de un doctorado curricular en estomatología. Revista Cubana de Estomatología, 48(3), 301-314.

Esta obra está bajo una Licencia Creative Commons Attribución-NoCommercial 4.0 International

(c) BY-NC 